Original paper

\title{
Influence of ursodeoxycholic acid therapy on levels of fibroblast growth factor 21 , adiponectin and biochemical parameters in intrahepatic cholestasis of pregnancy
}

\author{
Michał Kukla1,2, Rafał Kierach³, Magdalena Skonieczna ${ }^{4,5}$, Dorota Hudy ${ }^{4,5}$, Krzysztof Dąbrowski³ ${ }^{3}$, Tomasz Menżyk ${ }^{6}$, \\ Aleksandra Derra ${ }^{7}$, Dariusz Boroń ${ }^{8,9,10}$, Beniamin Oskar Grabarek ${ }^{8,10}$ \\ 'Department of Gastroenterology and Hepatology, School of Medicine in Katowice, Medical University of Silesia in Katowice, Poland \\ 2Department of Endoscopy, University Hospital in Cracow, Poland \\ ${ }^{3}$ Gynecology and Obstetrics Ward District Railway Hospital in Katowice, Poland \\ ${ }^{4}$ Department of Systems Biology and Engineering, Silesian University of Technology, Gliwice, Poland \\ ${ }^{5}$ Biotechnology Centre, Silesian University of Technology, Gliwice, Poland \\ ${ }^{6}$ Department of Internal Medicine, Gastroenterology and Acute Intoxication, Regional Hospital, Tarnów, Poland \\ ${ }^{7}$ Department of Neurology, Medical Centre of Upper Silesia, Katowice, Poland \\ ${ }^{8}$ Department of Histology, Cytophysiology and Embryology in Zabrze, University of Technology, Faculty of Medicine, Katowice, Poland \\ ${ }^{9}$ Department of Gynecology and Obstetrics with Gynecologic Oncology, Ludwik Rydygier Memorial Specialized Hospital, Kraków, Poland \\ ${ }^{10}$ District Hospital in Chrzanow, Poland
}

\begin{abstract}
The aim was to assess whether fibroblast growth factor 21 (FGF-21) and adiponectin influence intrahepatic cholestasis of pregnancy (ICP) pathogenesis and whether ursodeoxycholic acid (UDCA) has an impact on their levels. 50 pregnant women with ICP (ICP PW), 50 with uncomplicated pregnancy (HPW) and 50 healthy nonpregnant women (HW) were included. In ICP PW the first blood sample was drawn at the time of diagnosis, while in HPW it was drawn in the $28^{\text {th }}$ week of pregnancy. The next blood samples were drawn in the $32^{\text {nd }}$ and $36^{\text {th }}$ week of pregnancy and one day after delivery. UDCA was administered when ICP was diagnosed. In ICP PW serum FGF-21 concentration was the lowest at the time of diagnosis with an evident increase after UDCA administration. Serum FGF-21 levels were significantly higher in ICP PW than in HPW from the first to the last measurement. There was a negative association between adiponectin and bile acids (BAs) levels in the later stage of pregnancy in ICP PW. Up-regulated FGF-21 serum levels in ICP patients compared to HPW persisted after delivery, suggesting its role in disease pathophysiology. The negative association between serum adiponectin and BAs of the later stage of pregnancy may suggest its role in regulation of BAs concentration. UDCA exerts a beneficial effect on insulin sensitivity and up-regulates FGF-21 in ICP.
\end{abstract}

Key words: adiponectin, fibroblast growth factor 21, insulin resistance, intrahepatic cholestasis of pregnancy, ursodeoxycholic acid, bile acids.

Address for correspondence:

Dr hab. n. med. Michał Kukla, Department of Gastroenterology and Hepatology, School of Medicine in Katowice, Medical University of Silesia in Katowice, Poland, e-mail: kuklamich@poczta.onet.pl

\section{Introduction}

Intrahepatic cholestasis of pregnancy (ICP), also known as obstetric cholestasis or hepatosis gestationa- lis, is the most common pregnancy-specific liver disease and the second most frequent cause of jaundice in pregnancy following viral hepatitis. This reversible form of cholestasis is characterized by otherwise unexplained 
pruritus present in the late second or third trimester and an elevated serum total bile acids (TBAs) level, as well as abnormal liver tests. All of the symptoms and signs usually spontaneous resolve rapidly after the delivery of the fetus $[1,2]$. Depending on the ethnicity and geographic location the frequency of ICP varies remarkably between $0.1 \%$ and $25 \%$. It is observed in $0.4-1 \%$ of pregnancies in most areas of Europe, with a higher incidence in Scandinavian countries ( $1.5 \%$ in Sweden). The frequency of ICP is also higher in South Asian (0.8-1.46\%) and South American populations [2-4]. The etiology of ICP is still not completely explicit; most of the publications emphasize the complex and multifactorial character of this condition. Hepatitis $\mathrm{C}$ virus (HCV) infection, advanced age, multiple gestations, and low selenium and vitamin $\mathrm{D}$ levels have been demonstrated as risk factors of ICP in several studies $[3,5]$. It is suggested that pathogenesis includes a combination of hormonal and environmental factors superimposing on a genetic predisposition. The maternal course of ICP is mostly benign, except an aggravating pruritus, without any severe long-term complications. Although it does not present a direct threat to the mother's life, it is associated with the possibility of chronic placental insufficiency. ICP is associated with increased risk of fetal complications, such as intrauterine fetal distress, preterm labor and sudden intrauterine fetal demise. Furthermore, it can lead to increased frequency of meconium staining of amniotic fluid, cardiotocography (CTG) abnormalities and respiratory distress syndrome (RDS) $[3,6,7]$. An early and accurate diagnosis with an appropriate medical intervention is mandatory for an improved fetal prognosis. As a first line treatment to reduce maternal pruritus and decrease laboratory abnormalities ursodeoxycholic acid (UDCA) is recommended in a dose of $10-15 \mathrm{mg} / \mathrm{kg} /$ day [8].

Fibroblast growth factor 21 (FGF-21), a peptide hormone and a member of the FGF superfamily, has emerged as an important endocrine metabolic regulator with physiological effects on glucose and lipid metabolism. It is mainly produced in the liver and in smaller amounts by several metabolically active tissue organs, such as adipose tissue, skeletal muscle, and pancreas [9, 10]. Fasting and starvation are the main factors increasing expression of FGF-21 in plasma and liver under the control of the nuclear receptor peroxisome proliferator-activated receptor $\alpha$ (PPAR $\alpha$ ) [11-13]. FGF-21 regulates the energy balance through both the central nervous system (CNS) control of food intake and adaptation of hepatic, adipose tissue, and possibly pancreatic metabolism to fasting-feeding periods. If FGF-21 expression is stimulated under fasting conditions; it induces hepatic gluconeogenesis [13]. Several studies have demonstrated metabolic benefits of administered systemically FGF-21, which include weight loss, improvement in glucose and lipid metabolism, and insulin sensitivity. FGF-21 therapy has been shown to increase brown adipocyte numbers, preserve $\beta$-cell function and mass, ameliorate hepatic steatosis, reduce low-density lipoprotein cholesterol (LDL-C), and elevate high-density lipoprotein cholesterol (HDL-C) $[9,12]$. Fasting plasma levels of FGF-21 are low in healthy subjects. Increased levels of FGF-21 are observed commonly in diseases characterized by the accumulation of ectopic fat in the liver and dysregulation of adipose tissue metabolism $[14,15]$.

Adiponectin is a $30 \mathrm{kDa}$ multimeric protein produced and secreted almost exclusively by adipose tissue, but also expressed in other tissues including liver parenchyma cells, human osteoblasts, epithelial cells, myocytes and placental tissue [16]. In serum, this adipokine occurs in three forms: trimers, hexamers and high-molecular weight (HMW) multimers, which is the most biologically active isoform [17]. Adiponectin effects are mediated almost exclusively by adiponectin receptors, which occur as two isoforms (AdipoR1 and AdipoR2), but also another receptor has been identified for adiponectin, called T-cadherin. It acts as a receptor for hexameric and HMW forms of adiponectin but not for other forms $[16,18]$. Adiponectin demonstrates insulin-sensitizing, vascular-protective and anti-inflammatory properties [19]. Adiponectin strongly inhibits hepatic glucose production, acting locally in key metabolic tissues, promotes insulin sensitization, and enhances the oxidation of free fatty acids in skeletal muscles. It has a protective effect on the body's functioning by inhibiting cell death, decreasing inflammation and increasing cell survival [19].

Taking into account the main role of FGF-21 and adiponectin in the physiological process and pathophysiological condition, the aim of our study was to assess whether FGF-21 and adiponectin influence ICP pathogenesis and whether UDCA has an impact on their levels.

\section{Experimental section}

The study was performed on 50 pregnant women with ICP (ICP PW). The term ICP was used if the serum bile acids (BAs) level was $\geq 10 \mu \mathrm{mol} / \mathrm{l}$ with concomitant pruritus which could not be explained by any other conditions. Patients with ICP were aged 32.0 (28.25-34.0) years, with body mass index (BMI) 28.4 (24.26-30.1) $\mathrm{kg} / \mathrm{m}^{2}$. We compared the study group with 1) a group of 50 healthy, pregnant women without ICP (HPW), age 26.0 (21.0-30.0) years and BMI 22.1 (20.61-27.49) kg/m², and 2) a group of 50 healthy nonpregnant women (HW), age 31.5 (24.5-39.5) years and BMI $26.3(24.37-26.99) \mathrm{kg} / \mathrm{m}^{2}$. We excluded patients and healthy pregnant women with no permission for contribution in research, incomplete data, fetal con- 
genital anomalies, multiple pregnancies, HELLP syndrome (hemolysis, elevated liver enzymes, and a low platelet count syndrome), insulin dependent diabetes mellitus, malignancies, chronic heart failure, chronic kidney disease, psychiatric disorders, any alcohol consumption, or thyroid disorders. The exclusion criteria were also abnormal transaminases and cholestatic enzymes, viral hepatitides, symptomatic cholelithiasis, cholecystitis, primary sclerosing cholangitis (PSC), primary biliary cholangitis (PBC), autoimmune hepatitis (AIH), Wilson's disease, al-antitrypsin deficiency, cytomegalovirus or Epstein-Barr virus infection, acute fatty liver of pregnancy, drug-induced liver disease and HIV infection. Additional exclusion criteria for HPW were abnormal transaminases and cholestatic enzymes activities. The exclusion criteria for the group of $50 \mathrm{HW}$ were as mentioned above but also alcohol consumption of more than $20 \mathrm{~g} /$ day. The assessed clinical and laboratory parameters in all groups are presented in Table 1.

All parameters in pregnant women were measured at four time points in fasting condition. In ICP PW the first blood sample was drawn at the time of diagnosis. The next two blood samples were drawn in the $32^{\text {nd }}$ and $36^{\text {th }}$ week of pregnancy on UDCA treatment at the dose $13-15 \mathrm{mg} / \mathrm{kg}$ and the last one day after delivery. Among HPW parameters were measured at $28^{\text {th }}, 32^{\text {nd }}$ and $36^{\text {th }}$ week of pregnancy, while the last blood sample was drawn one day after the labor. In HW the blood sample was taken once.

The remaining biochemical parameters were measured using routine methods. The upper limit of normal (ULN) for ALT activity was set at $38 \mathrm{IU} / \mathrm{l}$ and for AST at $40 \mathrm{IU} / \mathrm{l}$. Insulin resistance (IR) was calculated according to the homeostasis model assessment for IR (HOMA-IR) by the formula: fasting insulin level $(\mathrm{mUI} / \mathrm{l}) \times$ fasting glucose level $(\mathrm{mg} / \mathrm{dl}) / 405$.

The study was approved by the Ethical Committee of the Silesian Medical University in Katowice No. KNW/0022/KB1/45/I/15, dated 29.09.2015 and conformed to the ethical guidelines of the Declaration of Helsinki. Informed consent was obtained for the whole study series.

The statistical analysis was performed with STATISTICA 10.0 (StatSoft Polska Sp. z o.o., Cracow, Poland). The data were expressed as median (25\% quartile- $75 \%$ quartile) or mean \pm standard deviation. The Shapiro-Wilk test was used to evaluate the distribution. The statistical significance of the difference in studied variables was tested using the Mann-Whitney $U$-test and ANOVA rank Kruskal-Wallis tests for independent groups. Correlations were analyzed with the Spearman rank correlation coefficient. Statistical significance was defined as values of $p<0.05$.

\section{Results}

\section{Baseline characteristics and laboratory data of ICP PW, HPW and HW}

The baseline characteristics and laboratory data of the study group ICP PW, HPW and HW after first measurement are shown in Table 1.

\section{Comparison of FGF-21, adiponectin, BAs levels and other laboratory parameters at different time points in ICP PW}

Serum FGF-21 concentration was significantly lower at the time of diagnosis compared to the last measurement [0.18 (0.15-0.48) vs. $17.3(0.49-50.22) \mathrm{ng} / \mathrm{ml}, p=0.04)$. There was however no significant increase in FGF-21 levels after UDCA administration in the $32^{\text {nd }}$ and $36^{\text {th }}$ week compared to the first measurement (Table 2). No significant difference was observed in serum adiponectin levels between subsequent measurements among ICP PW. Serum BAs levels decreased after UDCA introduction, but the difference reached statistical significance only between the first and third measurement [32.8 (24.43$45.5)$ vs. $21.0(16.15-31.03) \mu \mathrm{mol} / \mathrm{l}, p=0.04)$. Fasting insulin level was the highest at the time of diagnosis and was significantly lower in the next measurements [11.0 (5.75-16.60) vs. 7.77 (5.84-19.22) vs. 5.30 (4.99-6.03) vs. $5.32(4.98-7.80) \mathrm{mIU} / \mathrm{ml})$ in ICP PW group. Also HOMA-IR was the highest at the first time point with significant depletion in subsequent measurements after UDCA administration.

\section{Comparison between ICP PW and HPW at different time points}

Serum FGF-21 levels were significantly higher in ICP PW in the first, third and last measurement when compared the same time period in HPW. After UDCA administration the difference was even more visible and still statistically significant (Table 3 ). There was no difference in serum adiponectin concentration between ICP PW and HPW at any time point. Serum BAs levels were significantly higher in ICP PW in all measurements compared to HPW $(p<0.001, p<0.001, p=0.002$, $p=0.003$, respectively). The same findings were observed with respect to alanine transaminase (ALT) and aspartate transaminase (AST) activities (Table 3).

\section{Comparison between HW and ICP PW}

We also compared ICP PW with HW (Table 4). There was no difference in FGF-21 concentration be- 
Table 1. Baseline characteristics and laboratory data of ICP PW, HPW and HW after first measurement

\begin{tabular}{|c|c|c|c|c|c|c|}
\hline Parameter & $\begin{array}{c}\text { HW } \\
(n=20)\end{array}$ & $\begin{array}{l}\text { ICP PW } 1 \\
(n=26)\end{array}$ & $\begin{array}{l}\text { HPW } 1 \\
(n=20)\end{array}$ & $\begin{array}{c}\text { HW/ICP PW } \\
\text { (p-value) }\end{array}$ & $\begin{array}{c}\text { ICP PW/HPW } \\
\text { (p-value) }\end{array}$ & $\begin{array}{l}\text { HPW/HW } \\
\text { (p-value) }\end{array}$ \\
\hline Age (years) & $\begin{array}{c}31.5 \\
(24.5-39.5)\end{array}$ & $\begin{array}{c}32.0 \\
(28.25-34.0)\end{array}$ & $\begin{array}{c}26.0 \\
(21.0-30.0)\end{array}$ & 0.72 & 0.02 & 0.11 \\
\hline Waist girth (cm) & $\begin{array}{c}72.5 \\
(67.5-82.0)\end{array}$ & $\begin{array}{c}83.0 \\
(75.0-90.0)\end{array}$ & $\begin{array}{c}70.0 \\
(65.0-81.25)\end{array}$ & 0.09 & 0.07 & 0.76 \\
\hline $\mathrm{BMI}\left(\mathrm{kg} / \mathrm{m}^{2}\right)$ & $\begin{array}{c}26.3 \\
(24.37-26.99)\end{array}$ & $\begin{array}{c}28.4 \\
(24.26-30.1)\end{array}$ & $\begin{array}{c}22.1 \\
(20.61-27.49)\end{array}$ & 0.26 & 0.06 & 0.25 \\
\hline Bilirubin (mg/dl) & $\begin{array}{c}0.65 \\
(0.49-0.87)\end{array}$ & $\begin{array}{c}0.55 \\
(0.32-0.68)\end{array}$ & $\begin{array}{c}0.28 \\
(0.25-0.37)\end{array}$ & 0.30 & 0.12 & 0.01 \\
\hline Fasting insulin (mIU/ml) & $\begin{array}{c}6.30 \\
(5.46-8.34) \\
\end{array}$ & $\begin{array}{c}11.0 \\
(5.75-16.60)\end{array}$ & $\begin{array}{c}9.13 \\
(6.87-9.46) \\
\end{array}$ & 0.08 & 0.45 & 0.28 \\
\hline Fasting glucose (mg/dl) & $\begin{array}{c}78.8 \\
(69.37-94.86) \\
\end{array}$ & $\begin{array}{c}86.3 \\
(76.57-95.91) \\
\end{array}$ & $\begin{array}{c}104.0 \\
(89.39-105.26)\end{array}$ & 0.33 & 0.13 & 0.28 \\
\hline HOMA-IR & $\begin{array}{c}1.01 \\
(0.97-1.69)\end{array}$ & $\begin{array}{c}2.20 \\
(1.37-3.3)\end{array}$ & $\begin{array}{c}2.32 \\
(1.7-3.31)\end{array}$ & 0.04 & 0.79 & 0.01 \\
\hline Cholesterol (mg/dl) & $\begin{array}{c}185.0 \\
(169.0-201.0)\end{array}$ & $\begin{array}{c}312.0 \\
(271.5-318.25)\end{array}$ & $\begin{array}{c}301.5 \\
(263.5-307.0)\end{array}$ & $<0.001$ & 0.27 & 0.004 \\
\hline $\mathrm{HDL}(\mathrm{mg} / \mathrm{dl})$ & $\begin{array}{c}52.5 \\
(47.5-62.5) \\
\end{array}$ & $\begin{array}{c}75.0 \\
(69.5-83.0)\end{array}$ & $\begin{array}{c}82.5 \\
(76.5-90.0) \\
\end{array}$ & 0.002 & 0.28 & 0.01 \\
\hline LDL (mg/dl) & 100.5 & $\begin{array}{c}189.3 \\
(155.08-198.1)\end{array}$ & $\begin{array}{c}154.3 \\
(125.35-167.73) \\
\end{array}$ & 0.20 & 0.28 & 0.50 \\
\hline Triglycerides (mg/dl) & 68.0 & $\begin{array}{c}262.0 \\
(226.75-279.25)\end{array}$ & $\begin{array}{c}248.0 \\
(185.0-335.5) \\
\end{array}$ & 0.20 & 0.64 & 0.40 \\
\hline Iron $(\mu \mathrm{g} / \mathrm{dl})$ & $\begin{array}{c}63.9 \\
(40.58-80.63) \\
\end{array}$ & $\begin{array}{c}98.3 \\
(75.89-116.9) \\
\end{array}$ & $\begin{array}{c}120.4 \\
(93.7-183.53) \\
\end{array}$ & 0.07 & 0.50 & 0.02 \\
\hline $\operatorname{TIBC}(\mu \mathrm{g} / \mathrm{dl})$ & $\begin{array}{c}259.5 \\
(242.0-327.5) \\
\end{array}$ & $\begin{array}{c}473.5 \\
(446.0-499.0) \\
\end{array}$ & $\begin{array}{c}406.0 \\
(385.75-430.0)\end{array}$ & $<0.001$ & 0.05 & 0.08 \\
\hline $\mathrm{UIBC}(\mu \mathrm{g} / \mathrm{dl})$ & $\begin{array}{c}215.0 \\
(165.67-263.76)\end{array}$ & $\begin{array}{c}362.3 \\
(345.68-395.73)\end{array}$ & $\begin{array}{c}292.8 \\
(190.87-306.07)\end{array}$ & 0.004 & 0.02 & 0.78 \\
\hline $\mathrm{HGB}(\mathrm{mg} / \mathrm{dl})$ & $\begin{array}{c}12.6 \\
(12.0-12.95) \\
\end{array}$ & $\begin{array}{c}12.2 \\
(11.5-12.95)\end{array}$ & $\begin{array}{c}12.0 \\
(11.35-12.55) \\
\end{array}$ & 0.37 & 0.79 & 0.25 \\
\hline $\operatorname{RBC}\left(10^{6} / \mu \mathrm{l}\right)$ & $\begin{array}{c}4.12 \\
(4.01-4.33)\end{array}$ & $\begin{array}{c}3.96 \\
(3.84-4.3)\end{array}$ & $\begin{array}{c}3.80 \\
(3.72-4.11)\end{array}$ & 0.55 & 0.30 & 0.24 \\
\hline $\mathrm{WBC}\left(10^{6} / \mu \mathrm{l}\right)$ & $\begin{array}{c}11.3 \\
(8.29-11.51) \\
\end{array}$ & $\begin{array}{c}8.90 \\
(7.78-11.36) \\
\end{array}$ & $\begin{array}{c}9.14 \\
(7.98-11.41) \\
\end{array}$ & 0.51 & 0.95 & 0.81 \\
\hline $\operatorname{PLT}\left(10^{6} / \mu \mathrm{l}\right)$ & $\begin{array}{c}229.0 \\
(217.0-253.75)\end{array}$ & $\begin{array}{c}220.5 \\
(187.0-257.0) \\
\end{array}$ & $\begin{array}{c}224.5 \\
(168.5-272.0) \\
\end{array}$ & 0.58 & 0.93 & 0.93 \\
\hline Total protein (g/dl) & $\begin{array}{c}6.41 \\
(6.11-7.16) \\
\end{array}$ & $\begin{array}{c}6.60 \\
(6.5-6.75)\end{array}$ & $\begin{array}{c}6.40 \\
(6.33-6.56)\end{array}$ & 0.72 & 0.19 & 1.00 \\
\hline Albumin (g/dl) & 4.82 & $\begin{array}{c}3.74 \\
(3.62-3.99) \\
\end{array}$ & $\begin{array}{c}3.77 \\
(3.68-3.87)\end{array}$ & 0.29 & 0.91 & 0.40 \\
\hline Creatinine (mg/dl) & $\begin{array}{c}0.75 \\
(0.71-0.93)\end{array}$ & $\begin{array}{c}0.70 \\
(0.67-0.74)\end{array}$ & $\begin{array}{c}0.66 \\
(0.62-0.69)\end{array}$ & 0.13 & 0.18 & 0.06 \\
\hline $\mathrm{CRP}(\mathrm{mg} / \mathrm{l})$ & $\begin{array}{c}1.48 \\
(1.2-3.4)\end{array}$ & $\begin{array}{c}3.91 \\
(2.52-8.63)\end{array}$ & $\begin{array}{c}3.23 \\
(2.42-5.99)\end{array}$ & 0.07 & 0.75 & 0.15 \\
\hline
\end{tabular}

tween HW and the first measurement in ICP PW. However, FGF-21 concentrations after up- regulation at the next measurements in ICP PW appear to be significant- ly higher than in HW. There was no difference in adiponectin levels between HW and ICP PW at any time point. HOMA-IR was significantly higher in ICP PW 
Table 2. Comparison of laboratory parameters, HOMA-IR, FGF-21 and adiponectin levels at successive time points in ICP PW

\begin{tabular}{|c|c|c|c|c|c|c|}
\hline Parameter & ICP PW 1 & & ICP PW 2 & ICP PW 3 & & ICP PW 4 \\
\hline ALT (IU/I) & $\begin{array}{c}118.6 \\
(82.92-204.18)\end{array}$ & & $\begin{array}{c}114.4 \\
(32.99-133.62)\end{array}$ & $\begin{array}{c}131.3 \\
(32.53-172.85)\end{array}$ & & $\begin{array}{c}152.2 \\
(68.36-177.0)\end{array}$ \\
\hline AST (IU/I) & $\begin{array}{c}90.0 \\
(55.58-208.6)\end{array}$ & & $\begin{array}{c}65.5 \\
(30.29-119.72)\end{array}$ & $\begin{array}{c}67.7 \\
(25.81-85.09)\end{array}$ & & $\begin{array}{c}72.9 \\
(36.47-106.34)\end{array}$ \\
\hline Bilirubin (mg/dl) & $\begin{array}{c}0.55 \\
(0.32-0.68)\end{array}$ & & $\begin{array}{c}0.41 \\
(0.31-0.56)\end{array}$ & $\begin{array}{c}0.46 \\
(0.38-0.59)\end{array}$ & & $\begin{array}{c}0.50 \\
(0.38-0.65)\end{array}$ \\
\hline Alkaline phosphatase (IU/I) & $\begin{array}{c}154.3 \\
(105.2-226.15)\end{array}$ & & $\begin{array}{c}151.1 \\
(116.78-201.52)\end{array}$ & $\begin{array}{c}167.2 \\
(127.7-238.01)\end{array}$ & & $\begin{array}{c}169.3 \\
(133.24-228.8)\end{array}$ \\
\hline GGTP (IU/I) & $\begin{array}{c}13.9 \\
(13.21-20.03) \\
\end{array}$ & & $\begin{array}{c}14.8 \\
(11.33-20.35) \\
\end{array}$ & $\begin{array}{c}12.0 \\
(9.04-16.57)\end{array}$ & & $\begin{array}{c}12.4 \\
(10.67-15.61) \\
\end{array}$ \\
\hline Fasting glucose (mg/dl) & $\begin{array}{c}86.3 \\
(76.57-95.91) \\
\end{array}$ & & $\begin{array}{c}90.4 \\
(86.77-100.47)\end{array}$ & $\begin{array}{c}79.0 \\
(71.69-85.65)\end{array}$ & & $\begin{array}{c}82.0 \\
(72.8-85.88)\end{array}$ \\
\hline Bile acids $(\mu \mathrm{mol} / \mathrm{l})$ & $\begin{array}{c}32.8 \\
(24.43-45.5)\end{array}$ & & $\begin{array}{c}25.0 \\
(19.04-31.55)\end{array}$ & $\begin{array}{c}21.0 \\
(16.15-31.03)\end{array}$ & & $\begin{array}{c}27.5 \\
(16.4-31.1)\end{array}$ \\
\hline Fasting insulin (mlU/ml) & $\begin{array}{c}11.0 \\
(5.75-16.60)\end{array}$ & & $\begin{array}{c}7.77 \\
(5.84-19.22)\end{array}$ & $\begin{array}{c}5.30 \\
(4.99-6.03)\end{array}$ & & $\begin{array}{c}5.32 \\
(4.98-7.80)\end{array}$ \\
\hline Adiponectin (ng/ml) & $\begin{array}{c}12.2 \\
(10.48-15.20)\end{array}$ & & $\begin{array}{c}12.0 \\
(9.23-16.90)\end{array}$ & $\begin{array}{c}19.3 \\
(13.05-22.45)\end{array}$ & & $\begin{array}{c}16.6 \\
(12.0-22.28)\end{array}$ \\
\hline FGF-21 (ng/ml) & $\begin{array}{c}0.18 \\
(0.15-0.48)\end{array}$ & & $\begin{array}{c}9.22 \\
(0.15-66.39) \\
\end{array}$ & $\begin{array}{c}3.96 \\
(0.21-16.88) \\
\end{array}$ & & $\begin{array}{c}17.3 \\
(0.49-50.22) \\
\end{array}$ \\
\hline HOMA-IR & $\begin{array}{c}2.20 \\
(1.37-3.3)\end{array}$ & & $\begin{array}{c}1.62 \\
(1.3-4.4)\end{array}$ & $\begin{array}{c}1.07 \\
(0.88-1.51)\end{array}$ & & $\begin{array}{c}1.21 \\
(0.87-1.7)\end{array}$ \\
\hline \multicolumn{7}{|c|}{ Comparison between subgroups among ICP PW ( $p$-value) } \\
\hline Parameter & $\begin{array}{l}\text { ICP PW 1/ } \\
\text { ICP PW } 2\end{array}$ & $\begin{array}{l}\text { ICP PW 1/ } \\
\text { ICP PW } 3\end{array}$ & $\begin{array}{l}\text { ICP PW 1/ } \\
\text { ICP PW } 4\end{array}$ & $\begin{array}{l}\text { ICP PW 2/ } \\
\text { ICP PW } 3\end{array}$ & $\begin{array}{l}\text { ICP PW 2/ } \\
\text { ICP PW } 4\end{array}$ & $\begin{array}{l}\text { ICP PW 3/ } \\
\text { ICP PW } 4\end{array}$ \\
\hline ALT (IU/I) & 0.36 & 0.45 & 0.81 & 0.55 & 0.22 & 0.51 \\
\hline AST (IU/I) & 0.31 & 0.07 & 0.29 & 0.36 & 0.87 & 0.68 \\
\hline Bilirubin (mg/dl) & 0.56 & 1 & 0.64 & 0.38 & 0.24 & 0.76 \\
\hline Alkaline phosphatase (IU/I) & 0.97 & 0.70 & 0.75 & 0.60 & 0.53 & 1 \\
\hline GGTP (IU/I) & 0.91 & 0.24 & 0.37 & 0.23 & 0.43 & 0.99 \\
\hline Fasting glucose (mg/dl) & 0.21 & 0.11 & 0.22 & 0.002 & 0.01 & 0.69 \\
\hline Bile acids $(\mu \mathrm{mol} / \mathrm{l})$ & 0.09 & 0.04 & 0.11 & 0.51 & 0.89 & 0.69 \\
\hline Fasting insulin (mIU/ml) & 0.10 & 0.01 & 0.01 & 0.02 & 0.09 & 0.76 \\
\hline Adiponectin (ng/ml) & 0.82 & 0.06 & 0.18 & 0.13 & 0.31 & 0.78 \\
\hline FGF-21 (ng/ml) & 0.21 & 0.09 & 0.04 & 0.73 & 0.73 & 0.37 \\
\hline HOMA-IR & 0.86 & 0.02 & 0.04 & 0.02 & 0.07 & 0.81 \\
\hline
\end{tabular}

at the first and second measurements. However, after a decrease of HOMA-IR in ICP PW as of the $36^{\text {th }}$ week the difference was not significant any more. Serum BAs concentration and ALT and AST activities were significantly higher in ICP PW than in HW.

\section{Comparison between HW and HPW}

Surprisingly, FGF-21 serum concentration was significantly higher in HW than in HPW. There was no difference in adiponectin serum concentration. HOMA-IR was significantly higher at the $28^{\text {th }}$ week of pregnancy, with a subsequent decrease in HPW. BAs levels in HPW although significantly lower than in ICP PW were still significantly higher than in $\mathrm{HW}$ (Table 5).

\section{Correlations between analyzed parameters}

In the study we found a significant negative correlation of adiponectin with BAs in the group of ICP 
Table 3. Comparison of laboratory parameters, HOMA-IR, FGF-21 and adiponectin levels between ICP PW and HPW at different time points

\begin{tabular}{|c|c|c|c|c|c|c|c|c|}
\hline Parameter & ICP PW 1 & HPW 1 & ICP PW 2 & HPW 2 & ICP PW 3 & HPW 3 & ICP PW 4 & HPW 4 \\
\hline $\operatorname{ALT}(\mathrm{IU} / \mathrm{I})$ & $\begin{array}{c}118.6 \\
(82.92-204.18)\end{array}$ & $\begin{array}{c}15.1 \\
(11.67-23.65)\end{array}$ & $\begin{array}{c}114.4 \\
(32.99-133.62)\end{array}$ & $\begin{array}{c}14.0 \\
(11.55-16.65)\end{array}$ & $\begin{array}{c}131.3 \\
(32.53-172.85) \\
\end{array}$ & $\begin{array}{c}13.2 \\
(11.49-15.25)\end{array}$ & $\begin{array}{c}152.2 \\
(68.36-177.0)\end{array}$ & $\begin{array}{c}12.0 \\
(10.99-13.79)\end{array}$ \\
\hline AST (IU/I) & $\begin{array}{c}90.0 \\
(55.58-208.6)\end{array}$ & $\begin{array}{c}23.0 \\
(19.32-25.11)\end{array}$ & $\begin{array}{c}65.5 \\
(30.29-119.72)\end{array}$ & $\begin{array}{c}21.6 \\
(19.86-23.5)\end{array}$ & $\begin{array}{c}67.7 \\
(25.81-85.09)\end{array}$ & $\begin{array}{c}21.6 \\
(20.26-24.04)\end{array}$ & $\begin{array}{c}72.9 \\
(36.47-106.34)\end{array}$ & $\begin{array}{c}20.5 \\
(20.26-22.41)\end{array}$ \\
\hline $\begin{array}{l}\text { Bilirubin } \\
\text { (mg/dl) }\end{array}$ & $\begin{array}{c}0.55 \\
(0.32-0.68)\end{array}$ & $\begin{array}{c}0.28 \\
(0.25-0.37)\end{array}$ & $\begin{array}{c}0.41 \\
(0.31-0.56)\end{array}$ & $\begin{array}{c}0.34 \\
(0.28-0.42)\end{array}$ & $\begin{array}{c}0.46 \\
(0.38-0.59)\end{array}$ & $\begin{array}{c}0.42 \\
(0.39-0.45)\end{array}$ & $\begin{array}{c}0.50 \\
(0.38-0.65)\end{array}$ & $\begin{array}{c}0.44 \\
(0.4-0.49)\end{array}$ \\
\hline $\begin{array}{l}\text { Alkaline } \\
\text { phosphatase } \\
\text { (IU/I) }\end{array}$ & $\begin{array}{c}154.3 \\
(105.2-226.15)\end{array}$ & $\begin{array}{c}105.9 \\
(82.66-110.74)\end{array}$ & $\begin{array}{c}151.1 \\
(116.78-201.52)\end{array}$ & $\begin{array}{c}121.6 \\
(87.63-137.33)\end{array}$ & $\begin{array}{c}167.2 \\
(127.7-238.01)\end{array}$ & $\begin{array}{c}143.2 \\
(110.42-192.85)\end{array}$ & $\begin{array}{c}169.3 \\
(133.24-228.8)\end{array}$ & $\begin{array}{c}192.9 \\
(157.88-230.82)\end{array}$ \\
\hline GGTP (IU/I) & $\begin{array}{c}13.9 \\
(13.21-20.03) \\
\end{array}$ & $\begin{array}{c}9.79 \\
(7.43-28.54) \\
\end{array}$ & $\begin{array}{c}14.8 \\
(11.33-20.35) \\
\end{array}$ & $\begin{array}{c}9.16 \\
(7.07-24.86) \\
\end{array}$ & $\begin{array}{c}12.0 \\
(9.04-16.57) \\
\end{array}$ & $\begin{array}{c}8.68 \\
(6.38-10.71) \\
\end{array}$ & $\begin{array}{c}12.4 \\
(10.67-15.61) \\
\end{array}$ & $\begin{array}{c}8.68 \\
(6.6-11.98) \\
\end{array}$ \\
\hline $\begin{array}{l}\text { Fasting glucose } \\
(\mathrm{mg} / \mathrm{dl})\end{array}$ & $\begin{array}{c}86.3 \\
(76.57-95.91)\end{array}$ & $\begin{array}{c}104.0 \\
(89.39-105.26)\end{array}$ & $\begin{array}{c}90.4 \\
(86.77-100.47)\end{array}$ & $\begin{array}{c}83.5 \\
(83.01-84.64)\end{array}$ & $\begin{array}{c}79.0 \\
(71.69-85.65)\end{array}$ & $\begin{array}{c}87.3 \\
(81.75-92.26)\end{array}$ & $\begin{array}{c}82.0 \\
(72.8-85.88)\end{array}$ & $\begin{array}{c}88.6 \\
(78.28-100.57)\end{array}$ \\
\hline $\begin{array}{l}\text { Bile acids } \\
(\mu \mathrm{mol} / \mathrm{l})\end{array}$ & $\begin{array}{c}32.8 \\
(24.43-45.5) \\
\end{array}$ & $\begin{array}{c}7.40 \\
(5.05-9.75)\end{array}$ & $\begin{array}{c}25.0 \\
(19.04-31.55) \\
\end{array}$ & $\begin{array}{c}7.5 \\
(6.75-9.6)\end{array}$ & $\begin{array}{c}21.0 \\
(16.15-31.03) \\
\end{array}$ & $\begin{array}{c}9.50 \\
(7.023-10.45) \\
\end{array}$ & $\begin{array}{c}27.5 \\
(16.4-31.1)\end{array}$ & $\begin{array}{c}7.10 \\
(6.7-9.53)\end{array}$ \\
\hline $\begin{array}{l}\text { Fasting insulin } \\
(\mathrm{mll} / \mathrm{ml})\end{array}$ & $\begin{array}{c}11.0 \\
(5.75-16.60) \\
\end{array}$ & $\begin{array}{c}9.13 \\
(6.87-9.46) \\
\end{array}$ & $\begin{array}{c}7.77 \\
(5.84-19.22) \\
\end{array}$ & $\begin{array}{c}5.92 \\
(5.33-5.96) \\
\end{array}$ & $\begin{array}{c}5.30 \\
(4.99-6.03)\end{array}$ & $\begin{array}{c}6.92 \\
(5.5-8.46) \\
\end{array}$ & $\begin{array}{c}5.32 \\
(4.98-7.80) \\
\end{array}$ & $\begin{array}{c}7.29 \\
(6.22-8.43)\end{array}$ \\
\hline $\begin{array}{l}\text { Adiponectin } \\
(\mathrm{ng} / \mathrm{ml})\end{array}$ & $\begin{array}{c}12.2 \\
(10.48-15.20) \\
\end{array}$ & $\begin{array}{c}15.7 \\
(11.8-19.0) \\
\end{array}$ & $\begin{array}{c}12.0 \\
(9.23-16.90) \\
\end{array}$ & $\begin{array}{c}25.8 \\
(12.58-30.27) \\
\end{array}$ & $\begin{array}{c}19.3 \\
(13.05-22.45) \\
\end{array}$ & $\begin{array}{c}18.1 \\
(10.67-19.82) \\
\end{array}$ & $\begin{array}{c}16.6 \\
(12.0-22.28) \\
\end{array}$ & $\begin{array}{c}19.55 \\
(9.60-21.39) \\
\end{array}$ \\
\hline $\begin{array}{l}\text { FGF-21 } \\
(\mathrm{ng} / \mathrm{ml})\end{array}$ & $\begin{array}{c}0.18 \\
(0.15-0.48)\end{array}$ & $\begin{array}{c}0.13 \\
(0.11-0.15) \\
\end{array}$ & $\begin{array}{c}9.22 \\
(0.15-66.39) \\
\end{array}$ & $\begin{array}{c}0.15 \\
(0.15-0.15) \\
\end{array}$ & $\begin{array}{c}3.96 \\
(0.21-16.88) \\
\end{array}$ & $\begin{array}{c}0.15 \\
(0.15-0.15)\end{array}$ & $\begin{array}{c}17.3 \\
(0.49-50.22) \\
\end{array}$ & $\begin{array}{c}0.15 \\
(0.15-0.15) \\
\end{array}$ \\
\hline HOMA-IR & $\begin{array}{c}2.20 \\
(1.37-3.3) \\
\end{array}$ & $\begin{array}{c}2.32 \\
(1.7-3.31) \\
\end{array}$ & $\begin{array}{c}1.62 \\
(1.3-4.4) \\
\end{array}$ & $\begin{array}{c}1.13 \\
(1.11-1.21)\end{array}$ & $\begin{array}{c}1.07 \\
(0.88-1.51)\end{array}$ & $\begin{array}{c}1.46 \\
(1.16-1.86)\end{array}$ & $\begin{array}{c}1.21 \\
(0.87-1.7) \\
\end{array}$ & $\begin{array}{c}1.50 \\
(1.29-1.77)\end{array}$ \\
\hline \multicolumn{9}{|c|}{ Comparison between ICP PW and HPW (p-value) } \\
\hline Parameter & \multicolumn{2}{|c|}{ ICP PW 1/HPW 1} & \multicolumn{2}{|c|}{ ICP PW $2 / H P W ~ 2$} & \multicolumn{2}{|c|}{ ICP PW $3 /$ HPW 3} & \multicolumn{2}{|c|}{ ICP PW 4/HPW 4} \\
\hline ALT (IU/I) & \multicolumn{2}{|c|}{0.01} & \multicolumn{2}{|c|}{$<0.001$} & \multicolumn{2}{|c|}{0.01} & \multicolumn{2}{|c|}{0.02} \\
\hline AST (IU/I) & \multicolumn{2}{|c|}{0.004} & \multicolumn{2}{|c|}{$<0.001$} & \multicolumn{2}{|c|}{0.02} & \multicolumn{2}{|c|}{0.11} \\
\hline $\begin{array}{l}\text { Bilirubin } \\
\text { (mg/dl) }\end{array}$ & \multicolumn{2}{|c|}{0.12} & \multicolumn{2}{|c|}{0.37} & \multicolumn{2}{|c|}{0.33} & \multicolumn{2}{|c|}{0.53} \\
\hline $\begin{array}{l}\text { Alkaline } \\
\text { phosphatase } \\
\text { (IU/I) }\end{array}$ & \multicolumn{2}{|c|}{0.10} & \multicolumn{2}{|c|}{0.24} & \multicolumn{2}{|c|}{0.54} & \multicolumn{2}{|c|}{0.61} \\
\hline GGTP (IU/I) & \multicolumn{2}{|c|}{0.30} & \multicolumn{2}{|c|}{0.43} & \multicolumn{2}{|c|}{0.06} & \multicolumn{2}{|c|}{0.19} \\
\hline $\begin{array}{l}\text { Fasting glucose } \\
(\mathrm{mg} / \mathrm{dl})\end{array}$ & \multicolumn{2}{|c|}{0.13} & \multicolumn{2}{|c|}{0.01} & \multicolumn{2}{|c|}{0.04} & \multicolumn{2}{|c|}{0.12} \\
\hline $\begin{array}{l}\text { Bile acids } \\
(\mu \mathrm{mol} / \mathrm{l})\end{array}$ & \multicolumn{2}{|c|}{$<0.001$} & \multicolumn{2}{|c|}{$<0.001$} & \multicolumn{2}{|c|}{0.002} & 0.0 & \\
\hline $\begin{array}{l}\text { Fasting insulin } \\
(\mathrm{mlU} / \mathrm{ml})\end{array}$ & 0. & & 0.10 & & 0.0 & & 0.2 & 28 \\
\hline $\begin{array}{l}\text { Adiponectin } \\
\text { (ng/ml) }\end{array}$ & 0.3 & & 0.2 & & 0.5 & & 0.9 & 91 \\
\hline FGF-21 (ng/ml) & 0.0 & & 0.0 & & 0.0 & & 0.0 & 03 \\
\hline HOMA-IR & 0.7 & & $<0.0$ & & 0.1 & & 0.3 & 35 \\
\hline
\end{tabular}

PW at the third and last measurement $(r=[-0.61]$, $p<0.05$ and $r=[-0.85], p<0.01$, respectively). Adiponectin and FGF-21 did not show any other statis- tically significant correlation with liver function tests, fasting insulin, blood sugar, HOMA-IR or lipid profile in any group. 
Table 4. Comparison between ICP PW and HW

\begin{tabular}{|c|c|c|c|c|c|}
\hline Parameter & HW & ICP PW 1 & ICP PW 2 & ICP PW 3 & ICP PW 4 \\
\hline $\operatorname{ALT}(\mathrm{IU} / \mathrm{I})$ & $12.5 \pm 2.21$ & $\begin{array}{c}118.6 \\
(82.92-204.18)\end{array}$ & $\begin{array}{c}114.4 \\
(32.99-133.62)\end{array}$ & $\begin{array}{c}131.3 \\
(32.53-172.85)\end{array}$ & $\begin{array}{c}152.2 \\
(68.36-177.0)\end{array}$ \\
\hline AST (IU/I) & $16.6 \pm 3.29$ & $\begin{array}{c}90.0 \\
(55.58-208.60)\end{array}$ & $\begin{array}{c}65.5 \\
(30.29-119.72)\end{array}$ & $\begin{array}{c}67.7 \\
(25.81-85.09) \\
\end{array}$ & $\begin{array}{c}72.9 \\
(36.47-106.34) \\
\end{array}$ \\
\hline Bilirubin (mg/dl) & $0.65 \pm 0.26$ & $\begin{array}{c}0.55 \\
(0.32-0.68)\end{array}$ & $\begin{array}{c}0.41 \\
(0.31-0.56)\end{array}$ & $\begin{array}{c}0.46 \\
(0.38-0.59)\end{array}$ & $\begin{array}{c}0.50 \\
(0.38-0.65)\end{array}$ \\
\hline Alkaline phosphatase (IU/I) & $54.8 \pm 6.70$ & $\begin{array}{c}154.3 \\
(105.2-226.15)\end{array}$ & $\begin{array}{c}151.1 \\
(116.78-201.52)\end{array}$ & $\begin{array}{c}167.2 \\
(127.7-238.01)\end{array}$ & $\begin{array}{c}169.3 \\
(133.24-228.8)\end{array}$ \\
\hline GGTP (IU/I) & $14.4 \pm 3.92$ & $\begin{array}{c}13.9 \\
(13.21-20.03)\end{array}$ & $\begin{array}{c}14.8 \\
(11.33-20.35)\end{array}$ & $\begin{array}{c}12.0 \\
(9.04-16.57)\end{array}$ & $\begin{array}{c}12.4 \\
(10.67-15.61)\end{array}$ \\
\hline Fasting glucose (mg/dl) & $78.8 \pm 18.5$ & $\begin{array}{c}86.3 \\
(76.57-95.91) \\
\end{array}$ & $\begin{array}{c}90.4 \\
(86.77-100.47)\end{array}$ & $\begin{array}{c}79.0 \\
(71.69-85.65)\end{array}$ & $\begin{array}{c}82.0 \\
(72.8-85.88)\end{array}$ \\
\hline Bile acids $(\mu \mathrm{mol} / \mathrm{l})$ & $3.20 \pm 0.67$ & $\begin{array}{c}32.8 \\
(24.43-45.5)\end{array}$ & $\begin{array}{c}25.0 \\
(19.04-31.55)\end{array}$ & $\begin{array}{c}21.0 \\
(16.15-31.03)\end{array}$ & $\begin{array}{c}27.5 \\
(16.4-31.1)\end{array}$ \\
\hline Fasting insulin (mlU/ml) & $6.30 \pm 2.47$ & $\begin{array}{c}11.0 \\
(5.75-16.60)\end{array}$ & $\begin{array}{c}7.77 \\
(5.84-19.22)\end{array}$ & $\begin{array}{c}5.30 \\
(4.99-6.03)\end{array}$ & $\begin{array}{c}5.32 \\
(4.98-7.80)\end{array}$ \\
\hline Adiponectin (ng/ml) & $17.6 \pm 9.53$ & $\begin{array}{c}12.2 \\
(10.48-15.20) \\
\end{array}$ & $\begin{array}{c}12.0 \\
(9.23-16.90)\end{array}$ & $\begin{array}{c}19.3 \\
(13.05-22.45)\end{array}$ & $\begin{array}{c}16.6 \\
(12.0-22.28)\end{array}$ \\
\hline FGF-21 (ng/ml) & $0.24 \pm 32.4$ & $\begin{array}{c}0.18 \\
(0.15-0.48)\end{array}$ & $\begin{array}{c}9.22 \\
(0.15-66.39)\end{array}$ & $\begin{array}{c}3.96 \\
(0.21-16.88)\end{array}$ & $\begin{array}{c}17.3 \\
(0.49-50.22)\end{array}$ \\
\hline HOMA-IR & $1.01 \pm 0.45$ & $\begin{array}{c}2.20 \\
(1.37-3.3)\end{array}$ & $\begin{array}{c}1.62 \\
(1.3-4.4)\end{array}$ & $\begin{array}{c}1.07 \\
(0.88-1.51)\end{array}$ & $\begin{array}{c}1.21 \\
(0.87-1.7)\end{array}$ \\
\hline \multicolumn{6}{|c|}{ Comparison between ICP PW and HW ( $p$-value) } \\
\hline Parameter & ICP PW 1/HW & ICP PW 2/HW & & ICP PW 3/HW & ICP PW 4/HW \\
\hline ALT (IU/I) & $<0.001$ & $<0.001$ & & 0.003 & 0.004 \\
\hline AST (IU/I) & $<0.001$ & $<0.001$ & & $<0.001$ & 0.003 \\
\hline Bilirubin (mg/dl) & 0.30 & 0.05 & & 0.13 & 0.33 \\
\hline Alkaline phosphatase (IU/I) & $<0.001$ & $<0.001$ & & $<0.001$ & $<0.001$ \\
\hline GGTP (IU/I) & 0.76 & 0.69 & & 0.51 & 0.52 \\
\hline Fasting glucose (mg/dl) & 0.33 & 0.06 & & 1.00 & 0.76 \\
\hline Bile acids ( $\mu \mathrm{mol} / \mathrm{l})$ & $<0.001$ & $<0.001$ & & $<0.001$ & $<0.001$ \\
\hline Fasting insulin (mIU/ml) & 0.08 & 0.24 & & 0.11 & 0.22 \\
\hline Adiponectin (ng/ml) & 0.67 & 0.57 & & 0.80 & 1.00 \\
\hline FGF-21 (ng/ml) & 0.55 & 0.52 & & 0.74 & 0.35 \\
\hline HOMA-IR & 0.04 & 0.03 & & 0.89 & 0.96 \\
\hline
\end{tabular}

\section{Discussion}

As mentioned before, FGF-21 has been suggested to be an important regulator of energy metabolism having beneficial effects on glucose and lipid metabolism. FGF-21 expression is altered in patients with metabolic diseases, including metabolic syndrome, obesity, or type 2 diabetes [19]. Not only pathological phenomena influence this adipokine expression as well as serum circulating level, but also it is modified by physiological condition such as pregnancy, which is probably associated with the attempt to maintain metabolic homeostasis. Research performed on a mouse model as well as among pregnant women showed that plasma FGF-21 was significantly increased among study groups in comparison to non-pregnant controls $[20,21]$. In contrast, the results of our study showed significantly lower levels of FGF-21 among HPW in comparison to HW. Moreover, these disparities were noticeable in subsequent measurements, even after delivery. According to our 
Table 5. Comparison between HW and HPW

\begin{tabular}{|c|c|c|c|c|c|}
\hline Parameter & HW & HPW 1 & HPW 2 & HPW 3 & HPW 4 \\
\hline ALT (IU/I) & $12.5 \pm 2.21$ & $\begin{array}{c}15.1 \\
(11.67-23.65)\end{array}$ & $\begin{array}{c}14.0 \\
(11.55-16.65)\end{array}$ & $\begin{array}{c}13.2 \\
(11.49-15.25)\end{array}$ & $\begin{array}{c}12.0 \\
(10.99-13.79)\end{array}$ \\
\hline AST (IU/I) & $16.6 \pm 3.29$ & $\begin{array}{c}23.0 \\
(19.32-25.11)\end{array}$ & $\begin{array}{c}21.6 \\
(19.86-23.5)\end{array}$ & $\begin{array}{c}21.6 \\
(20.26-24.04)\end{array}$ & $\begin{array}{c}20.5 \\
(20.26-22.41) \\
\end{array}$ \\
\hline Bilirubin (mg/dl) & $0.65 \pm 0.26$ & $\begin{array}{c}0.28 \\
(0.25-0.37)\end{array}$ & $\begin{array}{c}0.34 \\
(0.28-0.42)\end{array}$ & $\begin{array}{c}0.42 \\
(0.39-0.45)\end{array}$ & $\begin{array}{c}0.44 \\
(0.4-0.49)\end{array}$ \\
\hline Alkaline phosphatase (IU/I) & $54.8 \pm 6.70$ & $\begin{array}{c}105.9 \\
(82.66-110.74)\end{array}$ & $\begin{array}{c}121.6 \\
(87.63-137.33)\end{array}$ & $\begin{array}{c}143.2 \\
(110.42-192.85)\end{array}$ & $\begin{array}{c}192.9 \\
(157.88-230.82)\end{array}$ \\
\hline GGTP (IU/I) & $14.4 \pm 3.92$ & $\begin{array}{c}9.79 \\
(7.43-28.54) \\
\end{array}$ & $\begin{array}{c}9.16 \\
(7.07-24.86)\end{array}$ & $\begin{array}{c}8.68 \\
(6.38-10.71) \\
\end{array}$ & $\begin{array}{c}8.68 \\
(6.6-11.98) \\
\end{array}$ \\
\hline Fasting glucose (mg/dl) & $78.8 \pm 18.5$ & $\begin{array}{c}104.0 \\
(89.39-105.26)\end{array}$ & $\begin{array}{c}83.5 \\
(83.01-84.64) \\
\end{array}$ & $\begin{array}{c}87.3 \\
(81.75-92.26) \\
\end{array}$ & $\begin{array}{c}88.6 \\
(78.28-100.57)\end{array}$ \\
\hline Bile acids $(\mu \mathrm{mol} / \mathrm{l})$ & $3.20 \pm 0.67$ & $\begin{array}{c}7.40 \\
(5.05-9.75)\end{array}$ & $\begin{array}{c}7.5 \\
(6.75-9.6)\end{array}$ & $\begin{array}{c}9.50 \\
(7.023-10.45)\end{array}$ & $\begin{array}{c}7.10 \\
(6.7-9.53)\end{array}$ \\
\hline Fasting insulin (mIU/ml) & $6.30 \pm 2.47$ & $\begin{array}{c}9.13 \\
(6.87-9.46)\end{array}$ & $\begin{array}{c}5.92 \\
(5.33-5.96)\end{array}$ & $\begin{array}{c}6.92 \\
(5.5-8.46)\end{array}$ & $\begin{array}{c}7.29 \\
(6.22-8.43)\end{array}$ \\
\hline Adiponectin (ng/ml) & $17.6 \pm 9.53$ & $\begin{array}{c}15.7 \\
(11.8-19.0)\end{array}$ & $\begin{array}{c}25.8 \\
(12.58-30.27) \\
\end{array}$ & $\begin{array}{c}18.1 \\
(10.67-19.82) \\
\end{array}$ & $\begin{array}{c}19.55 \\
(9.60-21.39)\end{array}$ \\
\hline FGF-21 (ng/ml) & $0.24 \pm 32.4$ & $\begin{array}{c}0.13 \\
(0.11-0.15)\end{array}$ & $\begin{array}{c}0.15 \\
(0.12-0.17) \\
\end{array}$ & $\begin{array}{c}0.15 \\
(0.13-0.17)\end{array}$ & $\begin{array}{c}0.15 \\
(0.13-0.18)\end{array}$ \\
\hline HOMA-IR & $1.01 \pm 0.45$ & $\begin{array}{c}2.32 \\
(1.7-3.31)\end{array}$ & $\begin{array}{c}1.13 \\
(1.11-1.21)\end{array}$ & $\begin{array}{c}1.46 \\
(1.16-1.86)\end{array}$ & $\begin{array}{c}1.50 \\
(1.29-1.77)\end{array}$ \\
\hline \multicolumn{6}{|c|}{ Comparison between HW and HPW (p-value) } \\
\hline Parameter & HW/HPW 1 & HW/HPW 2 & & HW/HPW 3 & HW/HPW 4 \\
\hline ALT (IU/I) & 0.76 & 0.66 & & 0.64 & 0.91 \\
\hline AST (IU/I) & 0.05 & 0.03 & & 0.01 & 0.03 \\
\hline Bilirubin (mg/dl) & 0.01 & 0.01 & & 0.02 & 0.11 \\
\hline Alkaline phosphatase (IU/I) & 0.02 & 0.003 & & $<0.001$ & 0.01 \\
\hline GGTP (IU/I) & 0.37 & 0.52 & & 0.01 & 0.05 \\
\hline Fasting glucose (mg/dl) & 0.28 & 0.34 & & 0.30 & 0.33 \\
\hline Bile acids $(\mu \mathrm{mol} / \mathrm{l})$ & $<0.001$ & $<0.001$ & & 0.002 & 0.003 \\
\hline Fasting insulin (mlU/ml) & 0.45 & 0.10 & & 0.09 & 0.28 \\
\hline Adiponectin (ng/ml) & 0.37 & 0.21 & & 0.51 & 1.00 \\
\hline FGF-21 (ng/ml) & 0.03 & 0.06 & & 0.006 & 0.03 \\
\hline HOMA-IR & 0.79 & $<0.001$ & & 0.15 & 0.35 \\
\hline
\end{tabular}

results, serum FGF-21 concentration was significantly lower in ICP PW at the time of diagnosis compared to successive measurements. The increased FGF-21 level was stable from the $2^{\text {nd }}$ to $4^{\text {th }}$ measurement on UDCA treatment.

Formerly, FGF-21 was investigated as a serum marker of pregnancy related pathologies such as preeclampsia $(\mathrm{PE})$ or gestational diabetes mellitus (GDM). Stepan et al. [22] reported median maternal FGF-21 serum concentrations to be almost three times higher in PE patients as compared to healthy, age-matched pregnant women (309.6 vs. $105.2 \mathrm{ng} / \mathrm{l}$, $p<0.001)$. Similarly, GDM is associated with a significant increase of this hepatokine serum concentration [23]. That easily leads us to the question: is there a possibility that FGF-21 plays a role in ICP pathogenesis? Based on our knowledge, this study is the first to try to answer this question. According to the results, serum FGF-21 levels were significantly higher in patients with ICP compared with healthy pregnant women. To fully understand how this hepatokine may influence pathophysiology of ICP, it is essential to analyze step 


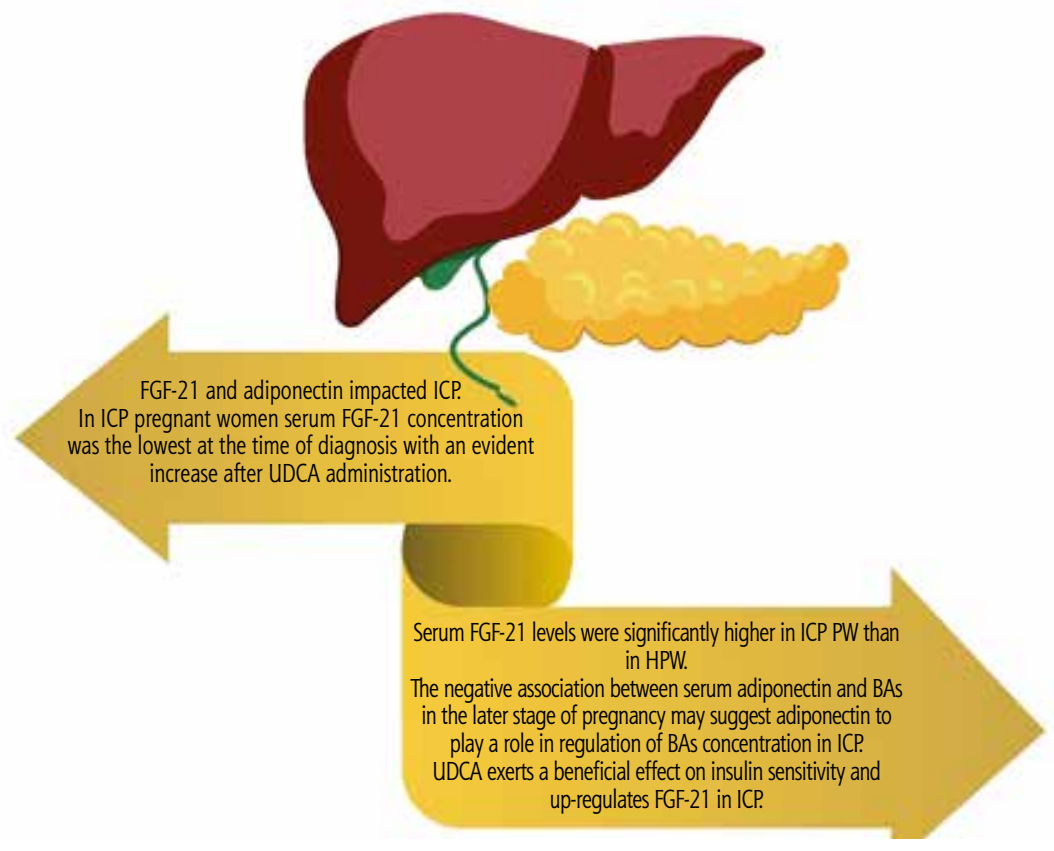

Fig. 1. The potential role of adiponectin and FGF-21 in intrahepatic cholestasis of pregnancy (ICP)

by step the mechanism of BAs synthesis and the role of FGF-21 in regulating that process. Zhang et al. [24] proved that overexpression of FGF-21 in mice leads to increased BAs synthesis and pool size. Further, the authors showed that adipokine regulates BAs metabolism indirectly by interfering with physiological inhibition of BAs synthesis via another member of the FGF subfamily, FGF-15/19 [24]. It is well established that FGF$15 / 19$ indirectly reduces the serum BAs concentration $[25,26]$. FGF-15/19 acts in the liver via the $\beta$ Klotho/ FGFR4 complex. Due to the competition between FGF$15 / 19$ and FGF-21 for access to $\beta$ Klotho, each increase of FGF-21 concentration reverses the inhibition of BAs synthesis by acting as an antagonist to FGF-15/19 function. The same study highlights the possible role of FGF-21 in BAs metabolism by its influence on the expression of hepatic and ileum membrane transporters, which play critical roles in maintaining BAs homeostasis. Nevertheless, this finding seems to be a secondary factor in increasing the circulating BAs pool size. According to all of the information presented above, we could suppose that significantly higher serum concentration of FGF-21 among ICP PW contrary to HPW in our research could be one of the main factors in ICP pathogenesis, since elevation of TBAs level is one of the diagnostic criteria of ICP. Furthermore, the fact that increased concentration of FGF-21 persisted after delivery suggests that elevation may not be just a transient, but rather an overall predisposition to cholestasis among ICP women.
Nonetheless, we have to emphasize the fact that there was no correlation between levels of FGF-21 and TBAs among the study group, inasmuch as a decrease of TBAs did not reflect the fluctuations of serum circulating FGF-21. If we analyze changes of serum BAs level, we could noticed the only significant difference in BAs concentration between first and third measurements without further decrease. If a trend test is performed and the result is significant, a trend can be reported. Otherwise, the description of a tendency remains unscientific. This tendency could be explained by treatment with UDCA, which was administered just after the diagnosis. UDCA has been proved to be effective for cholestatic diseases therapy, including ICP, not only due to the alleviation of pruritus, but also the improvement of biochemical liver tests and reduction of serum BAs levels $[19,27]$. According to several studies $[28,29]$ the reduction of BAs level is associated with the induction of key hepatobiliary transport proteins by UDCA. There is an evident difference between UDCA and FGF-21 with respect to the mechanism they play in BAs metabolism. While FGF-21 indirectly enhances synthesis of BAs, UDCA facilities their hepatobiliary transport. In view of the above information, the role of FGF-21 in the ICP pathogenesis cannot be excluded. The lack of correlation between adipokine and BAs levels among ICP PW could be explained by the deranging influence of UDCA treatment.

To the best of our knowledge this is the first study which takes into consideration the relation between 
ICP and adiponectin serum levels. Both ICP and changes in adiponectin expression and serum levels are related to metabolic disorders - dyslipidemia and glucose intolerance, first as a complication, and the latter as another factor playing a role in pathogenesis of these abnormalities.

In order to better understand the pathophysiology of metabolic disorders in ICP, firstly we have to retrace changes of glucose homeostasis in normal pregnancy. During normal pregnancy glucose metabolism and insulin production alter and lead to insulin resistance (IR), causing physiological maternal postprandial hyperglycemia. However, the mechanism responsible for IR has not been stated clearly. Some reports showed the correlation of longitudinal changes in IR with cytokines and adipokine concentration [30, 31]. Pregnancy-associated IR is reflected in higher HOMA-IR levels, which we can clearly observe also in the results of the present study. In comparison to HW, HOMA-IR was significantly higher at the $28^{\text {th }}$ week of pregnancy, with a subsequent decrease in HPW. An association between ICP and glucose intolerance and dyslipidemia has been previously reported. Previous studies have shown that the incidence of GDM is higher in women predisposed to developing ICP and proved the relation between these two $[32,33]$. A prospective study performed by Martineau et al. [33] on ICP patients showed significantly higher fasting total cholesterol, LDL cholesterol, serum triglycerides and reduced HDL cholesterol compared to those with uncomplicated pregnancy. Treatment with UDCA did not appear to have any influence on glucose or lipids in ICP women in this study. Despite the fact that Martineau's study indicates the lack of modification of glucose or lipid parameters via UDCA, some of the studies presented a potential effect on metabolic parameters. The meta-analysis performed by Sánchez-García et al. [34] revealed a significant reduction of fasting glucose levels following UDCA therapy. It also indicated a significant reduction of glycated hemoglobin $\left(\mathrm{HbA}_{1 \mathrm{c}}\right)$ concentrations. According to results presented in our study HOMA-IR was the highest at the first time point with significant depletion in subsequent measurements after UDCA administration. Simultaneously, fasting insulin level was the highest at the time of diagnosis and was significantly lower in the next measurements among ICP PW. These results may indicate the potential positive influence of UDCA treatment on IR. Furthermore, HOMA-IR was significantly higher in ICP $\mathrm{PW}$ than in $\mathrm{HW}$ at the first and second measurements. However, after the decrease of HOMA-IR in ICP PW as of the $36^{\text {th }}$ week the difference was not significant any more, which may also suggest a beneficial impact of
UDCA therapy. Although the underlying mechanisms by which UDCA ameliorates glucose metabolism are unclear, some of the researchers have tried to discover an explanation. It was proved that UDCA treatment decreases hepatic IR through reduction of hepatic glucose production, resulting in the improvement of hyperglycemia and hyperinsulinemia [34]. It also reduces serum levels of tumor necrosis factor $\alpha$ (TNF- $\alpha$ ), a pro-oxidant cytokine that promotes IR [35].

Hypoadiponectinemia is strongly correlated with occurrence of states associated with IR, including obesity, type 2 diabetes and GDM [36]. In obesity and type 2 diabetes, low plasma adiponectin concentrations are related to the degree of hyperinsulinemia and the severity of IR.

Pointing to the results of some studies which assessed serum adiponectin in pregnancy the interpretation of our results may be vague. Pregnancy is characterized by multiple modifications in the woman's physiology, including alteration in hormone and adipokine secretion. According to Catalano et al. [37] in uncomplicated pregnancy total plasma adiponectin was found to be decreased in late pregnancy compared with pre-gravid measurements. Additionally, progression of pregnancy was associated with a stepwise lowering of plasma adiponectin level, as it was found previously in mice [38]. Our results did not show any significant difference in adiponectin levels between ICP PW and HPW and HW. Moreover, there was no significant difference in adiponectin level in ICP PW at the time of disease onset and the later stage of pregnancy.

Up to now serum adiponectin has not been investigated in ICP. The study by Salman et al. [39] scrutinized secretion of adiponectin in cirrhotic patients and cirrhotic and cholestatic patients. The results proved that adiponectin level was significantly higher in cirrhotic patients in comparison to controls. Furthermore, this elevation was more pronounced in patients with intercurrent cirrhosis and cholestasis. Adiponectin level was also associated with laboratory markers of cholestasis (alkaline phosphatase, GGTP) and serum bilirubin level. In contrast to our study, Salman et al. found that adiponectin level is linked to the degree of hepatocellular injury and cholestasis. Additionally, adiponectin levels in cirrhosis were not related to parameters of body composition or metabolism but exclusively to reduced liver function. However, it is crucial to emphasize that the underlying etiology of liver cirrhosis and cholestasis in Salman's patients was chronic hepatitis $\mathrm{C}$, which potentially had a substantial influence on the disparities between the results. 
Similar conclusions to Salman's investigation were drawn from the study performed by Floreani et al. [40], which proved that adiponectin was significantly higher in patients with primary biliary cholangitis $(\mathrm{PBC})$ than in healthy controls. Another study by Tacke et al. [41] showed adiponectin to be increased in chronic liver disease of different etiology compared to controls. It is essential to note that patients with biliary or autoimmune chronic liver diseases had significantly higher adiponectin concentrations. Furthermore, the study also found a positive correlation between adiponectin and parameters indicating cholestasis, e.g. alkaline phosphatase, BAs, GGTP and bilirubin. These findings suggested that reduced biliary exertion of adiponectin in cholestatic liver disease may contribute to its increased concentrations in plasma.

Therefore this raises the question: what is the reason for the lack of significant adiponectin increase among ICP patients? Presumably, it is associated with different pathogenesis of cholestasis in ICP, development of which is connected with reduction of hepatic biliary transport proteins' expression and through internalization of the bile salt export pump (BSEP), and genetic defects in other canalicular transporters a cellular level [3] rather than the obstructive mechanism of cholestasis presented in the mentioned studies. However, another finding of our study is that serum adiponectin levels were negatively associated with $\mathrm{BAs}$ levels in the last two measurements in ICP PW. It may suggest adiponectin to play a role in down-regulation of BAs levels. However, this aspect must be confirmed in further studies.

The low number of patients may be considered a limitation of this study, but it enabled us to retain homogeneity of the compared groups, simultaneously. Moreover, the lack of GDM patients with ICP among the analyzed groups prevented us from assessing the influence of this factor on the levels of FGF-21 and especially adiponectin. Additionally, our study does not include the assessment of body composition, including fat mass, which imposes a negative impact on adiponectin concentration.

To the best of our knowledge, our data are the first to show up-regulated FGF-21 serum levels in ICP patients compared to healthy pregnant women, which may suggest the role of FGF-21 in the pathophysiology of ICP. Additionally, the fact that increased concentration of FGF-21 persisted after delivery suggests that the hepatokine may be a predisposing factor for ICP. On the other hand, the negative association between serum adiponectin and BAs in the later stage of pregnancy may suggest adiponectin to play a role in regulation of BAs concentration in ICP. UDCA exerts a beneficial effect on insulin sensitivity and up-regulates FGF-21 in ICP.

We believe that further, more complex research will enable to clarify the role of adiponectin and especially FGF-21 in the pathogenesis of ICP, which may have a positive impact on diagnosis and treatment of this condition in the future (Fig. 1).

\section{Disclosure}

The authors declare no conflict of interest.

\section{References}

1. Pusl T, Beuers U. Intrahepatic cholestasis of pregnancy. Orphanet J Rare Dis 2007; 2: 26.

2. Ozkan S, Ceylan Y, Ozkan OV, Yildirim S. Review of a challenging clinical issue: Intrahepatic cholestasis of pregnancy. World J Gastroenterol 2015; 21: 7134-7141.

3. Menżyk T, Bator M, Derra A, et al. The role of metabolic disorders in the pathogenesis of intrahepatic cholestasis of pregnancy. Clin Exp Hepatol 2018; 4: 217-223.

4. Geenes V, Williamson C. Intrahepatic cholestasis of pregnancy. World J Gastroenterol 2009; 15: 2049-2066.

5. Floreani A, Gervasi MT. New insights on intrahepatic cholestasis of pregnancy. Clin Liver Dis 2016; 20: 177-189.

6. Kowalska-Kanka A, Maciejewski T, Niemiec KT. The concentrations of bile acids and erythropoetin in pregnant women with intrahepatic cholestasis and the state of the fetus and newborn. Med Wieku Rozwoj 2013; 17: 232-245.

7. Arrese M, Reyes H. Intrahepatic cholestasis of pregnancy: a past and present riddle. Ann Hepatol 2006; 5: 202-205.

8. Bicoccaa MJ, Sperlingb JD, Chauhan SP. Intrahepatic cholestasis of pregnancy: review of six national and regional guidelines. Eur J Obstet Gynecol Reprod Biol 2018; 231: 180-187.

9. Fon Tacer K, Bookout AL, Ding X, et al. Comprehensive expression atlas of the fibroblast growth factor system in adult mouse. Mol Endocrinol 2010; 24: 2050-2064.

10. Xie T, Leung PS. Fibroblast growth factor 21: a regulator of metabolic disease and health span. Am J Physiol Endocrinol Metab 2017; 313: 292-302.

11. Galman C, Lundasen T, Kharitonenkov A, et al. The circulating metabolic regulator FGF21 is induced by prolonged fasting and PPARalpha activation in man. Cell Metab 2008; 8: 169-174.

12. Matikainen N, Taskinen MR, Stennabb S, et al. Decrease in circulating fibroblast growth factor 21 after an oral fat load is related to postprandial triglyceride-rich lipoproteins and liver fat. Eur J Endocrinol 2012; 166: 487-492.

13. Liang Q, Zhong L, Zhang J, et al. FGF21 maintains glucose homeostasis by mediating the cross talk between liver and brain during prolonged fasting. Diabetes 2014; 63: 4064-4075.

14. Arunkumar EA, Sushil KJ. Adiponectin, a therapeutic target for obesity, diabetes, and endothelial dysfunction. Int J Mol Sci 2017; 18: 1321.

15. Waluga M, Kukla M, Żorniak M, et al. Fibroblast growth factor-21 and omentin-1 hepatic mRNA expression and serum levels in morbidly obese women with non-alcoholic fatty liver disease. J Physiol Pharmacol 2017; 68: 363-374.

16. Robinson K, Prins J, Venkatesh B. Clinical review: Adiponectin biology and its role in inflammation and critical illness. Crit Care 2011; 15: 221. 
17. Hug C, Wang J, Ahmad NS, et al. T-cadherin is a receptor for hexameric and high-molecular-weight forms of Acrp30/adiponectin. Proc Natl Acad Sci USA 2004; 101: 10308-10313.

18. Wang ZV, Scherer PE. Adiponectin, the past two decades. J Mol Cell Biol 2016; 8: 93-100.

19. Zhang F, Yu L, Lin X, et al. Minireview: roles of fibroblast growth factors 19 and 21 in metabolic regulation and chronic diseases. Mol Endocrinol 2015; 29: 1400-1413.

20. Cui Y, Giesy SL, Hassan M, et al. Hepatic FGF21 production is increased in late pregnancy in the mouse. Am J Physiol Regul Integr Comp Physiol 2014; 307: 290-298.

21. Redondo-Angulo I, Mas-Stachurska A, Sitges M, et al. FGF21 is required for cardiac remodeling in pregnancy. Cardiovasc Res 2017; 113: 1574-1584.

22. Stepan H, Kley K, Hindricks J, et al. Serum levels of the adipokine fibroblast growth factor-21 are increased in preeclampsia. Cytokine 2013; 62: 322-326.

23. Yuan D, Wu BJ, Henry A, et al. Role of fibroblast growth factor 21 in gestational diabetes mellitus: A mini-review. Clin Endocrinol (Oxf) 2019; 90: 47-55.

24. Zhang J, Gupte J, Gong Y, et al. Chronic over-expression of fibroblast growth factor 21 increases bile acid biosynthesis by opposing FGF15/19 action. EbioMedicine 2017; 15: 173-183.

25. Holt JA, Luo G, Billin AN, et al. Definition of a novel growth factor-dependent signal cascade for the suppression of bile acid biosynthesis. Genes Dev 2003; 17: 1581-1591.

26. Inagaki T, Choi M, Moschetta A, et al. Fibroblast growth factor 15 functions as an enterohepatic signal to regulate bile acid homeostasis. Cell Metab 2005; 2: 217-225.

27. Beuers U. Drug insight: Mechanisms and sites of action of ursodeoxycholic acid in cholestasis. Nat Clin Pract Gastroenterol Hepatol 2006; 3: 318-328.

28. Zhang Y, Lu L, Victor DW, et al. Ursodeoxycholic acid and S-adenosylmethionine for the treatment of intrahepatic cholestasis of pregnancy: a meta-analysis. Hepat Mon 2016; 16: e38558.

29. Marschall HU, Wagner M, Zollner G, et al. Complementary stimulation of hepatobiliary transport and detoxification systems by rifampicin and ursodeoxycholic acid in humans. Gastroenterology 2005; 129: 476-485.

30. Yamashita H, Yasuhi I, Fukuda M, et al. The association between maternal insulin resistance in mid-pregnancy and neonatal birthweight in uncomplicated pregnancies. Endocr J 2014; 61: 1019-1024

31. Sotoodeh Jahromi A, Sanie MS, Yusefi A, et al. Association of tumor growth factor- $\beta$ and interferon- $\gamma$ serum levels with insulin resistance in normal pregnancy. Glob J Health Sci 2015; 8: 25-32.

32. Baliutavičienė D, Zubruvienè N, Zalinkevičius R. Pregnancy outcome in cases of intrahepatic cholestasis of pregnancy. Int J Gynaecol Obstet. 2011; 112: 250-251.

33. Martineau MG, Raker C, Powrie R, Williamson C. Intrahepatic cholestasis of pregnancy is associated with an increased risk of gestational diabetes. Eur J Obstet Gynecol Reprod Biol 2014; 176: 80-85.

34. Sánchez-García A, Sahebkar A, Simental-Mendía M, Simental-Mendía LE. Effect of ursodeoxycholic acid on glycemic markers: A systematic review and meta-analysis. Pharmacol Rep 2014; 135: 144-149.

35. Ratziu V, de Ledinghen V, Oberti F, et al. A randomized controlled trial of high-dose ursodesoxycholic acid for nonalcoholic steatohepatitis. J Hepatol 2011; 54: 1011-1019.

36. Weyer C, Funahashi T, Tanaka S, et al. Hypoadiponectinemia in obesity and type 2 diabetes: close association with insulin resis- tance and hyperinsulinemia. J Clin Endocrinol Metab 2001; 86: 1930-1935.

37. Catalano PM, Hoegh M, Minium J, et al. Adiponectin in human pregnancy: implications for regulation of glucose and lipid metabolism. Diabetologia 2006; 49: 1677.

38. Kondo E, Sugiyama T, Kusaka H, Toyoda N. Adiponectin mRNA levels in parametrial adipose tissue and serum adiponectin levels are reduced in mice during late pregnancy. Horm Metab Res 2004; 36: 465-469.

39. Salman TA, Allam N, Azab GI, et al. Study of adiponectin in chronic liver disease and cholestasis. Hepatol Int 2010; 4: 767774.

40. Floreani A, Variola A, Niro G, et al. Plasma adiponectin levels in primary biliary cirrhosis: a novel perspective for link between hypercholesterolemia and protection against atherosclerosis. Am J Gastroenterol 2008; 103: 1959-1965.

41. Tacke F, Wüstefeld T, Horn R, et al. High adiponectin in chronic liver disease and cholestasis suggests biliary route of adiponectin excretion in vivo. J Hepatol 2005; 42: 666-673. 